\title{
External beam irradiation of myocardial carcinoid metastases: a
} case report Jonathan Strosberg*, Sarah Hoffe, Todd Hazelton and Larry Kvols

Address: H. Lee Moffitt Cancer Center and Research Institute, 12902 Magnolia Drive, Tampa, FL 33612, USA

Email: Jonathan Strosberg* - jonathan.strosberg@moffitt.org; Sarah Hoffe - sarah.hoffe@moffitt.org;

Todd Hazelton - todd.hazelton@moffitt.org; Larry Kvols - larry.kvols@moffitt.org

* Corresponding author

Published: 19 September 2007

Journal of Medical Case Reports 2007, I:95 doi:10.1 186/1752-1947-1-95
Received: 15 May 2007

Accepted: 19 September 2007

This article is available from: http://www.jmedicalcasereports.com/content/I/I/95

(c) 2007 Strosberg et al; licensee BioMed Central Ltd.

This is an Open Access article distributed under the terms of the Creative Commons Attribution License (http://creativecommons.org/licenses/by/2.0), which permits unrestricted use, distribution, and reproduction in any medium, provided the original work is properly cited.

\begin{abstract}
The heart is an exceedingly rare site of metastatic involvement in carcinoid tumors. Only nineteen cases have been described in the literature over the past 30 years. We report here on a patient who presented with progressive carcinoid syndrome despite surgical resection of her liver metastases. She was found to have cardiac metastases on inidium-III-pentetreotide scintigraphy and subsequently underwent external beam radiation to the heart resulting in symptomatic palliation of her syndrome and objective radiographic response. To our knowledge, this is the first reported case of metastatic cardiac carcinoid treated with external beam irradiation.
\end{abstract}

\section{Case presentation}

A 45 year-old woman with a history of metastatic carcinoid disease presented to our institution with progressive flushing and diarrhea.

She had been diagnosed with a carcinoid tumor 18 months earlier when an enlarged mesenteric lymph node was discovered incidentally during laparoscopic gastric bypass surgery. Pathologic evaluation demonstrated a moderately differentiated neuroendocrine tumor. Laboratory studies revealed an elevation in 24 hour urine 5 hydroxyindoleacetic acid (5HIAA) of $37.4 \mathrm{mg}$ (reference range $1-6 \mathrm{mg}$ ) and an elevated serum serotonin of 1671 $\mathrm{ng} / \mathrm{ml}$ (reference range $22-180 \mathrm{ng} / \mathrm{ml}$ ). At the time of initial diagnosis, the patient denied any prior history of flushing, diarrhea or wheezing.

Magnetic resonance imaging of the abdomen revealed two hepatic masses, one in the dome of the liver measuring $6.8 \mathrm{~cm}$, and another in the posterior right hepatic lobe measuring $2.2 \mathrm{~cm}$. Indium-111-pentetreotide scintigra- phy (OctreoScan ${ }^{\mathrm{TM}}$ ) revealed a focus of activity in the right hepatic lobe, as well as two foci of intense activity in the cardiac region (figure 1). A primary gastrointestinal or bronchial tumor was not detected.

Depot octreotide therapy was initiated, and a series of three laparoscopic hepatic radiofrequency ablations were performed over the ensuing 6 months. Although this therapy resulted in normalization of the 24-hour urine 5HIAA, serum serotonin remained elevated $(539 \mathrm{ng} / \mathrm{ml})$. Moreover, the patient began developing progressive diarrhea and intense facial flushing despite dose escalation of depot octreotide from $30 \mathrm{mg}$ to $60 \mathrm{mg}$ every 4 weeks.

Repeat indium-111-pentetreotide scintigraphy twelve months after initial diagnosis demonstrated resolution of activity in the liver. However, persistent radiotracer uptake was detected in the region of the myocardium. Echocardiography was ordered to evaluate for the presence of cardiac metastases. It demonstrated trace to mild tricuspid 


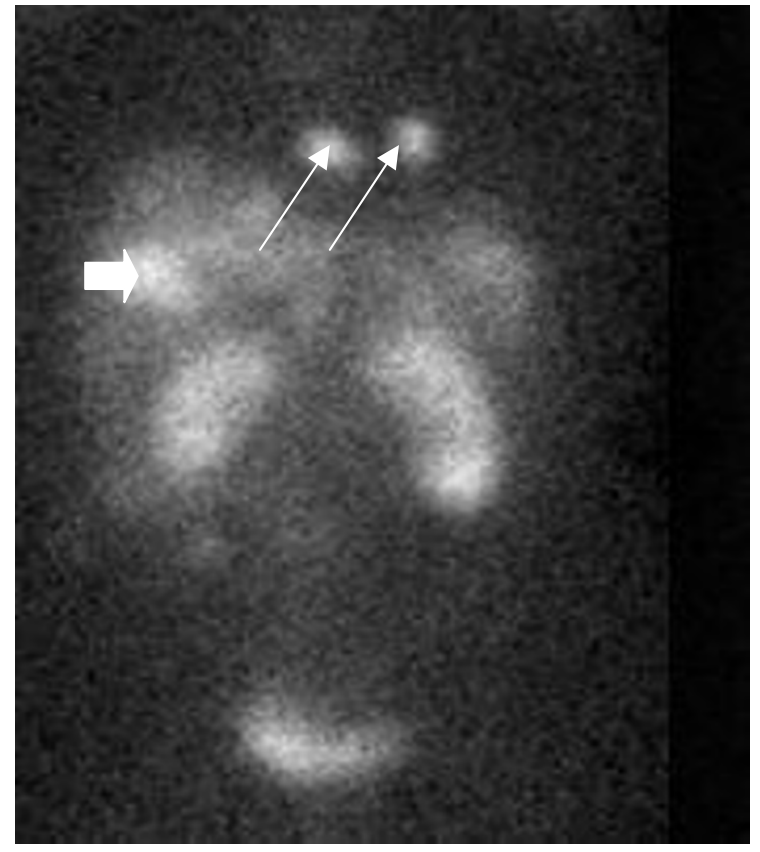

\section{Figure I}

In-I I I-Pentetriotide Scan (OctreoScan) demonstrating a liver metastasis (block arrow) as well as two distinct tumors in the region of the heart (thin arrows).

insufficiency and trace aortic insufficiency, but showed no evidence of an intracardiac mass.

Magnetic resonance (MR) imaging of the chest was performed using multiplanar breath-hold, electrocardiogram-gated HASTE (Half Fourier Single Shot Turbo SpinEcho) and electrocardiogram-gated, post-gadolinium T1weighted spin-echo images obtained on a 1.5-Tesla Symphony MR scanner (Siemens Medical Systems, Malvern, PA). The MR images demonstrated a $3.8 \times 2.1 \mathrm{~cm}$ mildly enhancing mass involving the free wall of the right ventricle as well as a poorly-defined mass involving the mid anterior left ventricular wall with extenstion to the apex. (Figure 2). These masses correlated to sites of abnormal radiotracer accumulation on indium-111-pentetreotide scintigraphy (Figure 2 inset).

Consideration was given to surgical resection of the myocardial metastases, however, the risk of malignant arrhythmias was deemed prohibitive. Therefore, radiation oncology was consulted for external beam irradiation. A total of 45 Gray was delivered to the myocardium in 25 fractions using 3D-conformal technique (figure 3 ). The patient's gross tumor volume (GTV) in both the right and left ventricles was drawn on the planning CT scans that

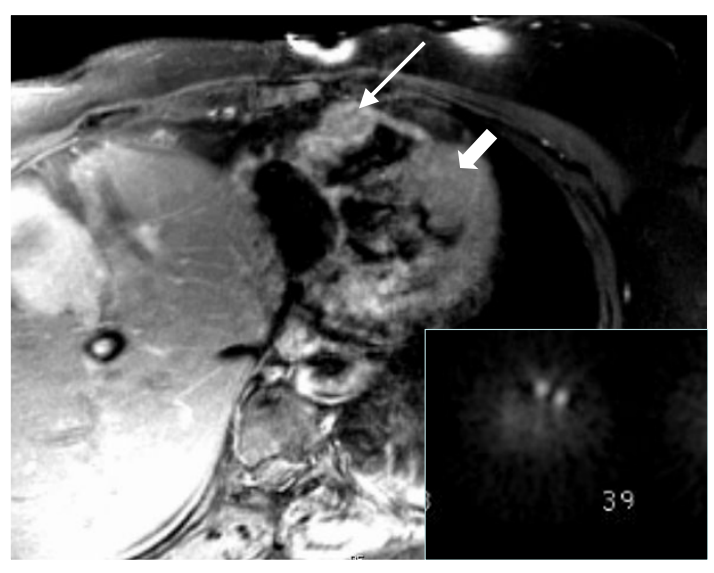

\section{Figure 2}

TI gated axial MRI image demonstrating mass in the right ventricular wall (long arrow) and more poorly-defined lesion in the left ventricular wall (block arrow) corresponding to sites of abnormal radiotracer uptake on indium- I I I-pentetreotide scintigraphy (inset).

had been taken with the patient in the supine position with her arms immobilized over her head in a vac-lok bag. The MRI images were used for aid in target volume delineation. Four beams consisting of an anterior superior oblique (ASO), anterior inferior oblique (AIO), left anterior superior oblique (LASO), and a right anterior superior oblique (RASO) were planned to deliver 45 Gy to the $\mathrm{GTV}+$ a $2 \mathrm{~cm}$ margin (figure 4). Dose volume histogram (DVH) analysis showed that with this beam arrangement, $<40 \%$ of the heart received the prescribed dose of $45 \mathrm{~Gy}$, $60 \%$ of the heart received $30 \mathrm{~Gy}$, and $50 \%$ received $40 \mathrm{~Gy}$. $30 \%$ of the total lung volume received $20 \mathrm{~Gy} .100 \%$ of the GTV received the prescription dose. Treatment was deliv-

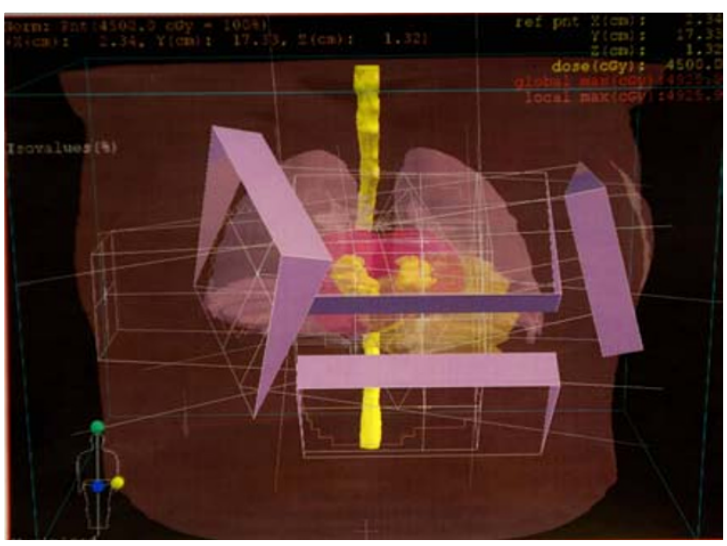

Figure 3

3-dimensional representation of fields with wedges. 


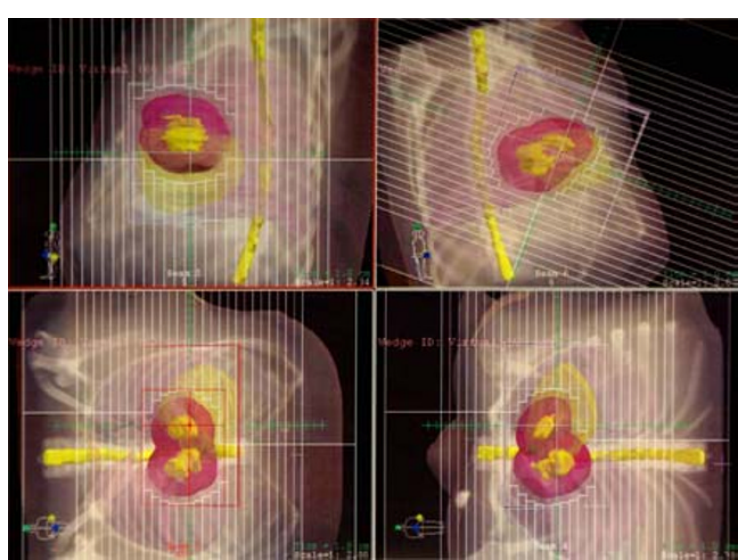

Figure 4

Multileaf collimator treatment fields.

ered using $18 \mathrm{MV}$ photons with 60 degree wedges on each field. The therapy was complicated only by a brief episode of chest pain and dyspnea without electrocardiographic changes.

Repeat magnetic resonance imaging after completion of radiation demonstrated a fifty percent bidimensional reduction in the size of the right ventricular tumor (figure 5 ) associated with a substantial decrease in serum serotonin levels to $205 \mathrm{ng} / \mathrm{ml}$. Six months after treatment, the patient denied any chest pain, dyspnea or orthopnea and reported a moderate improvement in her facial flushing and diarrhea. Interferon $\alpha$ was added to octreotide therapy for further management of her metastatic disease.

\section{Discussion}

Although right sided heart valve fibrosis and dysfunction is a hallmark of carcinoid heart disease, myocardial metastases from carcinoid tumors are distinctly uncommon. Among 243 patients undergoing echocardiography for evaluation for carcinoid heart disease, sonographic evidence of cardiac tumor was observed in only 6 patients (2\%).[1] Another series of 132 patients undergoing echocardiograms for evaluation of carcinoid heart disease demonstrated a $4 \%$ rate of myocardial metastases.[2]

While echocardiography has been the primary technique for identifying myocardial metastases from carcinoid tumors, other methods have also been described in the literature. Puvaneswary et al. described the use of magnetic resonance imaging for localization of cardiac metastases[3], while Yeung et al. reviewed the use of ${ }^{111}$ Indiumpentetreotide scintigraphy.[4] In this case, scintigraphy demonstrated radiotracer uptake in the region of the heart while MRI scans provided important anatomic information enabling use of focused radiation therapy. Echocardiography, however, failed to accurately identify the cardiac tumors.

The location of cardiac metastases in prior reported cases has included all chambers of the heart as well as pericardium. $[1,2,5,6]$ Two patients presented with malignant arrhythmias, including one patient who developed fatal bradycardia due to tumor infiltration of the atrioventricular node.[7] Carcinoid syndrome associated with elevation in urine 5HIAA has been documented in the majority of cases described in the literature.

An interesting feature of this case was the persistence of elevated serotonin along with progressive flushing and diarrhea despite the successful resection of liver metastases through radiofrequency ablation and normalization of urine 5 hydroxyindolacetic acid (5HIAA). Since inactivation of serotonin and its catabolism into 5HIAA occurs partially in the lung, we hypothesize that the direct secre-
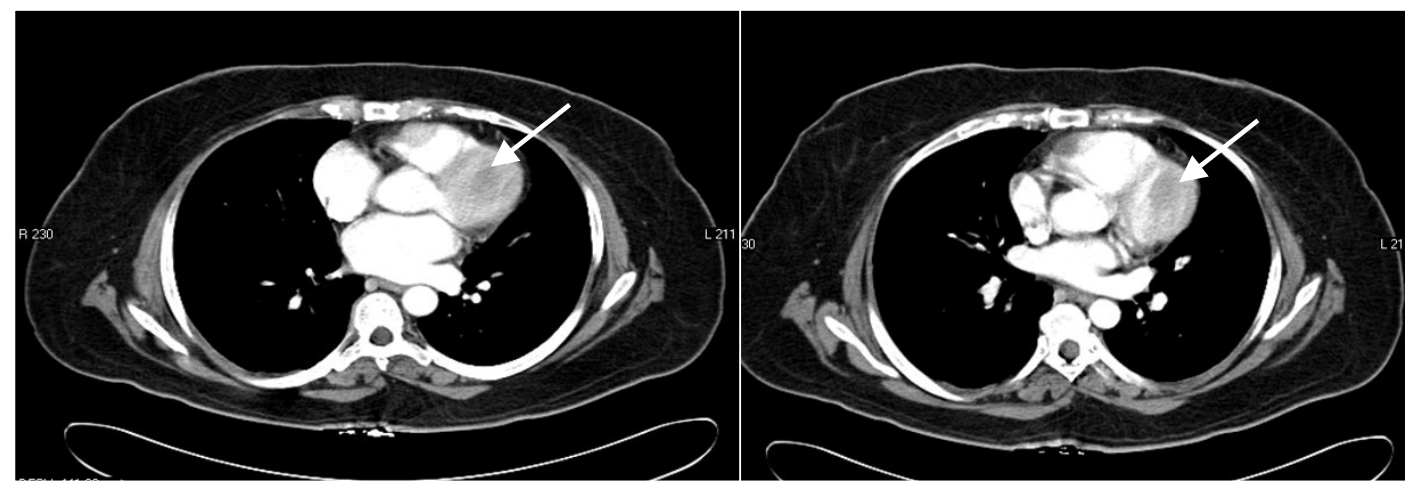

Figure 5

Left ventricular mass before (5.a) and after (5.b) external beam irradiation. 
tion of serotonin into the systemic circulation by the left ventricular metastasis may have resulted in exacerbation of the carcinoid syndrome despite ablation of liver metastases.

Surgical resection of cardiac metastases has been the therapy of choice in the majority of carcinoid tumors described in the literature. $[1,2,4]$ A minority of cases were treated with systemic chemotherapy.[5] The use of external beam irradiation for control of cardiac metastases from carcinoid tumors has not been described in the literature but there have been single institutional series reporting the effectiveness of radiation for other sites of metastatic disease.

Schupak and Wallner reviewed 44 patients with metastatic or unresectable carcinoid tumors who were treated at Memorial Sloan-Kettering Cancer Center with radiation.[8] The sites included in their series were epidural space, brain, bone, and abdomen. Infield control following radiotherapy was $77 \%$ for epidural sites and $78 \%$ for bone sites. No patient with brain metastasis had progression of intracranial disease after radiation and $80 \%$ of patients with abdominal disease achieved a complete or partial response. The authors did not demonstrate a dose response relationship and recommended 45-50 Gy in 45 weeks for non-hepatic sites. Chakravarthy and Abrams reported on 18 patients with metastatic or unresectable carcinoid patients at Johns Hopkins.[9] In their series, clinical improvement occurred in $87 \%$ of the sites irradiated.

Cardiac effects of radiation have been evaluated in great detail, with data derived largely from follow-up of long term survivors of Hodgkin's disease. Current evidence has shown that radiation to the heart can be associated with pericardial disease, accelerated atherogenesis of the coronary arteries, myocardial disease, valve defects, and predisposition to conduction abnormalities.[10-13]

\section{Conclusion}

The heart is a rare site of involvement in metastatic carcinoid tumors. Electrocardiogram-gated MRI may be a useful imaging modality for anatomic delineation of the tumor. Carcinoid tumors involving the myocardium can produce substantial morbidity due to invasion of sensitive cardiac structures and also due to secretion of serotonin directly into the systemic circulation. When surgical resection is considered excessively risky, external beam irradiation may be an effective option for palliation of symptoms. The potential benefits need to be weighed against the risks of pericardial, valvular and myocardial toxicity as well as chronic atherogenesis.

\section{Competing interests}

The author(s) declare that they have no competing interests.

\section{Authors' contributions}

SH commented on radiation technique and safety. TH commented on radiologic modalities and interpretation. JS and LK prepared the remainder of the manuscript including clinical history and therapeutic outcome.

\section{Acknowledgements}

Written patient consent was obtained for publication of this report.

\section{References}

I. Pandya UH, Pellikka PA, Enriquez-Sarano M, Edwards WD, Schaff HV, Connolly HM: Metastatic carcinoid tumor to the heart: echocardiographic-pathologic study of II patients. J Am Coll Cardiol 2002, 40(7): | 328- | 332.

2. Pellikka PA, Tajik AJ, Khandheria BK, Seward JB, Callahan JA, Pitot HC, Kvols LK: Carcinoid heart disease. Clinical and echocardiographic spectrum in $\mathbf{7 4}$ patients. Circulation 1993, 87(4): I $188-1 \mid 196$

3. Puvaneswary M, Thomson D, Bellamy GR: Cardiac metastasis from carcinoid tumour: magnetic resonance imaging findings. Australas Radiol 2004, 48(3):383-387.

4. Yeung HW, Imbriaco M, Zhang J], Macapinlac H, Goldsmith SJ, Larson SM: Visualization of myocardial metastasis of carcinoid tumor by indium-III-pentetreotide. I Nucl Med 1996, 37(9): $1528-1530$.

5. Drake WM, Jenkins PJ, Phillips RR, Lowe DG, Grossman AB, Besser GM, Wass JA: Intracardiac metastases from neuroendocrine tumours. Clin Endocrinol (Oxf) 1997, 46(4):5 I7-522.

6. Rich LL, Lisa CP, Nasser WK: Carcinoid pericarditis. Am J Med 1973, 54(4):522-527.

7. Shehata BM, Thomas JE, Doudenko-Rufforny I: Metastatic carcinoid to the conducting system-is it a rare or merely unrecognized manifestation of carcinoid cardiopathy? Arch Pathol Lab Med 2002, I 26(I 2): I538-1540.

8. Schupak KD, Wallner KE: The role of radiation therapy in the treatment of locally unresectable or metastatic carcinoid tumors. Int J Radiat Oncol Biol Phys I991, 20(3):489-495.

9. Chakravarthy A, Abrams RA: Radiation therapy in the management of patients with malignant carcinoid tumors. Cancer 1995, 75(6): 1386-1390.

10. Stewart JR, Fajardo LF: Dose response in human and experimental radiation-induced heart disease. Application of the nominal standard dose (NSD) concept. Radiology 197I, 99(2):403-408.

II. Carmel RJ, Kaplan HS: Mantle irradiation in Hodgkin's disease. An analysis of technique, tumor eradication, and complications. Cancer 1976, 37(6):2813-2825

12. Hancock SL, Tucker MA, Hoppe RT: Factors affecting late mortality from heart disease after treatment of Hodgkin's disease. Jama 1993, 270(16):1949-1955.

13. Adams MJ, Lipshultz SE, Schwartz C, Fajardo LF, Coen V, Constine LS: Radiation-associated cardiovascular disease: manifestations and management. Semin Radiat Oncol 2003, I3(3):346-356. 